Herzschr Elektrophys 2016 $27: 122-130$ DOI 10.1007/s00399-016-0432-8

Eingegangen: 4. April 2016

Angenommen: 12. April 2016

Online publiziert: 1. Juni 2016

(c) The Author(s) 2016. This article is available at SpringerLink with Open Access

CrossMark

\author{
Sabine Ernst ${ }^{1,2} \cdot$ Siew Yen $\mathrm{Ho}^{3} \cdot$ Karen McCarthy ${ }^{3}$ \\ ' Department of Cardiology, Royal Brompton and Harefield NHS Foundation Trust, London, UK \\ ${ }^{2}$ NIHR Cardiovascular Biomedical Research Unit, Royal Brompton and National Heart and Lung Institute, \\ Imperial College London, London, UK \\ ${ }^{3}$ Brompton Cardiac Morphology Unit, Imperial College London, London, UK
}

\title{
Arrhythmien bei Erwachsenen mit angeborenen Herzfehlern
}

\section{Atriale Tachykardien}

Patienten mit angeborenen Herzfehlern (AHF, mit und ohne Chirurgie des AHF) haben ein erhöhtes Risiko für sowohl Vorhof- als auch Kammerarrhythmien und den plötzlichen Herztod (SCD). Vor allem Vorhoftachykardien treten häufig auf und belasten die meist noch jungen Patienten sehr, die allein aufgrund der schnelleren Kammererregung und der uunter Umständen bereits in Sinusrhythmus limitierten Pumpfunktion schnell(er) hämodynamisch dekompensieren. Leider ist die Langzeittherapie mithilfe pharmakologischer Frequenzoder Rezidivkontrolle bei diesem Patientenkollektiv nicht sehr erfolgreich, sodass eine kurativ-intendierte Therapie (so sie denn gelingt) für diese Patienten viel attraktiver erscheint.

Die Behandlung durch Katheterablation ist in dieser relativ jungen Patientengruppe allerdings durch eine Reihe von Hindernissen erschwert:

- die zugrunde liegende Anatomie des ursprünglichen Herzfehlers (oder deren Kombination),

- die anatomischen Verhältnisse nach chirurgischer Korrekturoperation,

- die altersbedingte und durch etwaige chronisch-hämodynamische Überbelastung entstandene Fibrose oder Hypertrophie des Vorhofgewebes,

- das Vorliegen eines oder mehrerer Tachykardiemechanismen.

Selbst wenn diese genannten Hindernisse erfolgreich überwunden sind, kann sogar im Fall einer direkten Zugangsmöglichkeit die Erreichbarkeit der Zielregion und damit eventuell eine erfolgreiche
Ablation bereits durch die schiere Größe einer Herzkammer deutlich erschwert sein. Die Einführung von 3D-elektroanatomischen Mapping- und Navigationssystemen hat hier den entscheidenden Durchbruch der letzten Jahre gebracht, der sich in der deutlich verbesserten Erfolgsrate der Katheterablation von atrialen Tachykardien bei AHF niederschlägt [3].

Diese Übersichtsarbeit beschreibt die aktuell zur Verfügung stehenden technischen Verfahren und fasst die Erfolge bei der Behandlung der häufigsten atrialen Tachyarrhythmien zusammen.

\section{Vorhoftachykardiesubstrate}

Der häufigste Arrhythmiemechanismus bei Patienten mit AHF besteht aus einer Kreiserregung (Reentry-Tachykardie), die sich auf das Vorhofmyokard beschränkt. Diese sog. interoder intraatrialen Reentry-Tachykardien beschreiben Kreiserregungen um anatomische Hindernisse, z. B. Patch aus Goretex oder Dacron, chirurgische Nahtlinien nach Eröffnung der Vorhöfe, durch rechts- oder linksatriale Atriotomie oder als Folge der HerzLungen-Zirkulation während der Operation (Kanülierungsnarben), oder um die großen Atrioventrikularklappen herum (•Abb. 1). Detaillierte Kenntnisse der einzelnen Operationsverfahren und der etwaigen Verwendung von PatchMaterialien erlauben es, die potenziellen Erregungskreisläufe zu antizipieren. Allerdings gibt es auch vielen Patienten mit fokaler, d. h. von einer einzigen
Stelle ausgehenden, Tachykardie, deren Verständnis insbesondere in Gegenwart von großen Narbenarealen sehr schwierig sein kann. Außerdem haben AHF-Patienten sehr häufig mehr als einen Tachykardietyp und sorgfältige Kontrollstimulationen nach erfolgreicher Ablation der Indexarrhythmie sind notwendig, um weitere Arrhythmien nicht zu übersehen. Schlussendlich ist bei manchen Patienten mit AHF das Vorhofmyokard so sehr verdickt, dass eine tatsächlich transmurale, d. h. die gesamte Vorhofwand betreffende, Ablation schwierig oder gar unmöglich ist [27]. Das Gegenteil, mit z. T. extrem dünner Vorhofwand kann ebenfalls auftreten (• Abb. 1).

Detailliertes Mapping und kritische Überprüfung der 3D-Mapping-Daten mithilfe konventioneller Stimulationsmanöver sind essenziell für die erfolgreiche Durchführung einer Ablationsprozedur bei diesen komplexen Patienten.

\section{Verständnis der zugrunde liegenden Anatomie}

In den letzten Jahren hat sich die Verfügbarkeit und Qualität der 3D-Bildgebungstechniken durch Computertomographie (CT), Magnetresonanztomographie (MRT) und 3D-Echokardiographie dramatisch verbessert. Insbesondere bei komplexen AHF ist eine 3D-Rekonstruktion der individuellen Anatomie des jeweiligen Patienten sehr hilfreich (- Abb. 2; [24]). Diese erlaubt dem Untersucher, sich im Vorfeld der Prozedur nicht nur mit den Herzkammern, son- 

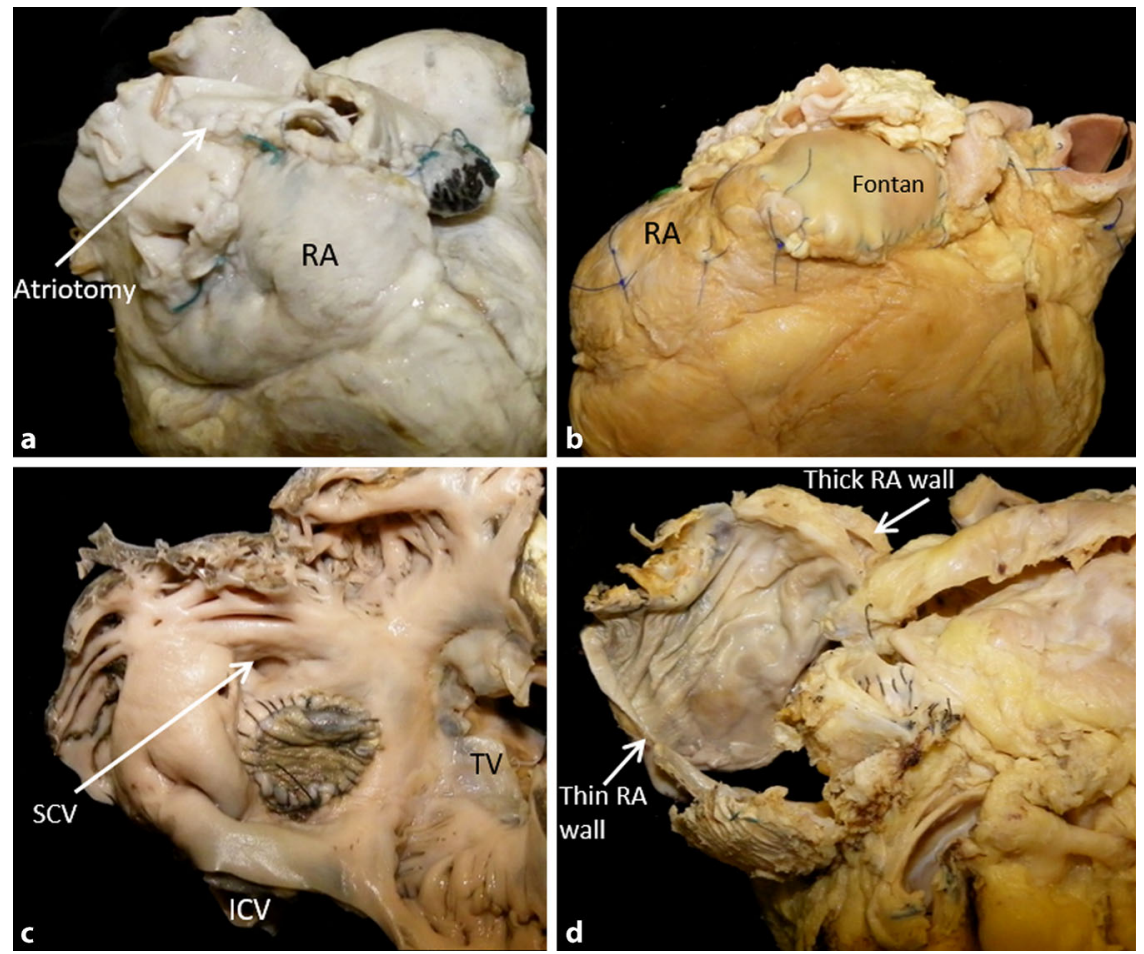

Abb. 1 A Anatomische Präparate. a Transposition der großen Gefäße mit atrialem Switch (MustardOperation); $\mathbf{b}$ klassischer RA-PA-Fontan mit großem Patch; $\mathbf{c}$ chirurgischer Vorhofseptumverschluss mit Patch; $\mathbf{d}$ Demonstration verschiedener Gewebedicken bei Z. n. modifizierter RA-PA-Fontan-Operation; ICV, RA rechter Vorhof, PA Pulmonalarterie, ICV inferiore Hohlvene, SCV superiore Hohlvene, TV Trikuspidalklappe

dern auch mit den zuführenden Gefäßen vertraut zu machen. Eine detaillierte Prozedurplanung und Wahl des optimalen Zugangswegs für den Mapping- und Ablationskatheter und etwaiger weitere diagnostischer Katheter kann so erfolgen (z. B. über eine V.-hemiazygosKontinuität bei angeborenem Verschluss der unteren Hohlvene; $[8,9]$ ). Das MRT besticht hier durch den Vorteil, dass bei Einsatz von sog. TrueFISP-Sequenzen keine Kontrastmittelgabe erfolgen muss, sondern Blut als intrinsisches Kontrastmittel dargestellt wird [13]. Dadurch wird die Gabe von großen Kontrastmittelboli (wie in der Kontrastmittel-CT erforderlich) vermieden, und es müssen keine Transitzeiten des Kontrastmittels durch das Herz berücksichtigt werden. Da beim MRT auch keine Strahlenbelastung anfällt, ist diese 3D-Bildgebung bei den meist jungen Patienten grundsätzlich vorzuziehen, solange keine eindeutigen Kontraindikationen bestehen (z. B. MRT-inkompatibler implantierbarer Kardioverter-Defibrillator oder Schrittmacher). Insbesondere bei Patimit blockierten Femoralgefäßen, kann der Bereich der MRT-Darstellung unbedenklich groß sein, um so die Optionen z. B. für einen superioren Zugangsweg wählen zu können. Die direkte Darstellung von Narbenarealen durch LateGadolinium-Enhancement(LGE)-MRT ist für Ventrikelmyokard bereits etabliert, aber für Vorhofmyokard noch in der Entwicklungsphase [2]. Die genaue Kenntnis der Dimensionen des Zielvorhofs erlaubt außerdem die geeigneten Instrumente auszuwählen, wie Katheter mit z. B. großem Kurvenradius oder zusätzlichen langen (steuerbaren) Schleusen. In manchen Fällen kann der Zugang zu der Zielregion durch Hindernisse wie z. B. künstliche Herzklappen oder BaffleMaterial versperrt sein. Dann müssen alternative Zugangswege durch transbaffle oder trans-hepatischer Punktion erwogen oder, falls vorhanden, die magnetische Navigation eingesetzt werden [7].

\section{Mapping}

\section{Dreidimensionale Systeme}

Mit der Einführung der 3D-elektroanatomischen Mappingsysteme erlebte die Ablation von Vorhoftachykardien bei Patienten mit AHF praktisch einen Quantensprung. So konnten jetzt erstmals die jeweiligen Herzkammern in 3D dargestellt und die bestehende, regelmäßige Tachykardie zeitlich abgebildet werden, was das Verständnis des Tachykardiemechanismus deutlich erleichterte. Zusätzlich kam es durch Einsatz von 3D-Mappingverfahren zu einer signifikanten Reduktion der Gesamtstrahlenbelastung und nicht zuletzt zu einer Verbesserung der Erfolgsraten für die Ablation bei AHF sowohl akut als auch langfristig [12, 21].

Die direkte Integration der prä-prozeduralen 3D-Bildgebung ist heutzutage Standard für alle zur Verfügung stehenden Mappingverfahren, was die Projektion der gemappten auf die dargestellten Herzkammer erleichtert und etwaige nicht erreichte Areale aufzeigt [6]. Zusätzlich erlaubt eine Bild-in-Bild-Integration auf die Standardröntgenprojektion eine weitere Verbesserung der Orientierung (•Abb. 2).

\section{Sequenzielle versus simultane Verfahren}

In den letzten Jahren sind neue simultane Mappingverfahren vorgestellt worden, die entweder invasiv (mithilfe von Multielektroden-Basketkathetern) oder nichtinvasiv (mithilfe sog. Body-Surface-Elektrokardiogramme) in der Lage sind, mit einem einzigen Herzschlag die gesamte Erregungsausbreitung zu erfassen. Für die letztere Variante stehen jetzt Daten zur Behandlung von Patienten mit AHF zur Verfügung [10]. Bei diesem System zeichnen 252 Oberflächen-Elektrokardiographie-Elektroden in Kombination mit einem Nativ-CT des Thorax den jeweiligen Herzrhythmus auf und stellt diesen als 3D-Aktivierungssequenz auf die gemeinsame Vorhof- (oder Ventrikel-)Anatomie dar (• Abb. 3a). Das Verfahren bietet sich besonders bei Patienten mit multiplen Tachykardien oder nur spontan auftretenden Tachykardien 
an, da es als nichtinvasives Verfahren den diagnostischen Teil der elektrophysiologischen Untersuchung (EPU) auf die Station verlagert. Dort kann der Patient alle möglichen Provokationsmanöver ausprobieren (z. B. Fahrradergometrie, sonstige körperliche Aktivität, Nahrungsaufnahme, etc.) während das System über bis zu 8 Stunden jeden einzelnen Herzschlag aufzeichnet. Ist die klinische Arrhythmie aufgezeichnet, wird der Patient ins Katheterlabor gebracht und die jeweiligen Tachykardiesubstrate (Reentry- oder fokale Tachykardie) mithilfe konventioneller elektrophysiologischer Kriterien überprüft und anschließend behandelt.

In der Gruppe der sequenziellen Mappingsystem (•Abb. 3b, c) geht der Trend zum Multielektrodenmapping, das in kürzerer Zeit eine größere Anzahl von Mappingpunkten aufzeichnet [19, 26]. Dafür bedarf es jedoch einer stabilen Tachykardie, die für den Patienten ebenfalls ausreichend hämodynamisch tolerabel sein muss. Da ein direkter Kontakt zwischen Mappingelektrode(n) und Myokard erforderlich ist, besteht die Gefahr der mechanischen Terminierung oder Alteration der Tachykardie (in eine oder mehrere andere Tachykardien). Ein Beispiel des Rhythmia ${ }^{\text {Tm }}$-Systems (Boston Scientific, Saint Paul/MN, USA), das innerhalb kurzer Zeit einige tausend Mappingpunkte mithilfe eines kleinen Basketkatheters aufzeichnet, zeigt - Abb. 3c. Im Vergleich zu benachbarten Punkten wird so die Erregungsausbreitung während Tachykardie anhand von weiteren Kriterien (Zykluslängenstabilität, Voltage etc.) rekonstruiert und erlaubt eine schnelle 3D-Darstellung. Daten von größeren AHF-Kollektiven liegen jedoch bis jetzt noch nicht vor.

\section{Katheterablationstechniken}

Bereits die myokardioale Dicke der chronisch volumenbelasteten oder vernarbten Vorhofwand kann ein unüberwindbares Hindernis bei der Katheterablation von Patienten mit AHF darstellen, selbst dann wenn alle anderen Hilfsverfahren (3D-Mapping, 3D-Bildintegration etc.) und konventionelle elektrophysiologische Stimulationsmanöver das Tachy-

Herzschr Elektrophys 2016 · 27:122-130 DOI 10.1007/s00399-016-0432-8

( $)$ The Author(s) 2016. This article is available at SpringerLink with Open Access

\section{S. Ernst $\cdot$ S. Yen Ho $\cdot$ K. McCarthy}

\section{Arrhythmien bei Erwachsenen mit angeborenen Herzfehlern. Atriale Tachykardien}

\section{Zusammenfassung}

Atriale Arrhythmien sind bei Patienten mit angeborenen Herzfehlern (AHF) mit und ohne operative Korrektur sehr häufig, und können nur mit beschränkter Erfolgsrate pharmakologisch behandelt werden. Dieser Übersichtsbeitrag beschreibt die heutzutage zur Verfügung stehenden Technologien, um in dieser extrem schwierigen Patientengruppe erfolgreiche Katheterablationen durchzuführen. Neben dem Verständnis der zugrunde liegenden Anatomie, die durch 3DBildgebung mithilfe von Magnetresonanztomographie oder Computertomographie ergänzt wird, wird die Auswahl der 3DMappingverfahren (sequenziell vs. simultan) vorgestellt. Schließlich wird die konventionelle Navigationstechnik im Vergleich zur magnetischen Navigation erläutert und die jeweiligen atrialen Arrhythmien in Bezug auf die verschiedenen AHF diskutiert.

\section{Schlüsselwörter}

Diagnostische Bildgebung · Computerunterstützte Bildinterpretation - Katheterablation . Schnittanatomie $\cdot$ Dreidimensionale Bildgebung

\section{Arrhythmia in adults with congenital heart defects. Atrial tachycardia}

\section{Abstract}

Atrial arrhythmias are frequently encountered in patients with congenital heart disease (CHD) with or without corrective surgery and respond to pharmacological therapies with only limited success. This review describes the technologies currently available for performing successful ablation procedures in this very complex patient cohort. In addition to an understanding of the underlying anatomy, which can be supplemented by 3D imaging with the aid of magnetic resonance imaging (MRI) or computed tomography (CT), the choice between the different 3D mapping systems (sequential versus simultaneous) is presented. Finally, conventional manual navigation is compared with magnetic navigation and then discussed with regard to the respective arrhythmias encountered with the different forms of CHD.

\section{Keywords}

Diagnostic imaging · Computer-assisted image interpretation - Catheter ablation . Cross-sectional anatomy - Three-dimensional imaging kardiesubstrat eindeutig definiert haben. Die Einführung sog. gekühlter („,irrigated tip“) Ablationskatheter, die durch konstante Spülung der Katheterspitze eine größere Eindringtiefe der Ablationsläsion erlauben, hat hier eine deutliche Verbesserung erbracht. Zusätzlich in die Katheterspitze integrierte Sensoren erlauben nun bei einigen Geräten den Anpressdruck des Mappingkatheters zu beurteilen, um so ebenfalls die Qualität der Ablationsläsion $\mathrm{zu}$ verbessern. Jedoch in Situationen mit geringem Katheter-Gewebe-Kontakt (z. B. bei massiv dilatierten Vorhöfen) oder bei erhöhtem oder reduziertem Blutfluss bleibt die Anlage permanenter und transmura- ler Ablationsläsionen immer noch eine Herausforderung [11].

\section{Ferngesteuerte Magnetfeld- navigation}

Das magnetische Navigationssystem Niobe $^{\circledR}$ (Stereotaxis Inc, St. Louis, USA) besteht aus 2 computergesteuerten Permanentmagneten (bestehend aus den Seltenen Erden Neodym, Bor und Eisen), die auf beiden Seiten des Kathetertischs positioniert werden und im Bereich des Thorax ein uniformes Magnetfeld mit einer Stärke von bis zu 0,1 T erzeugen. Ein sehr weicher und flexibler Katheter, der in seiner Spitze ebenfalls kleine Permanentmagnete aufweist, muss sich 


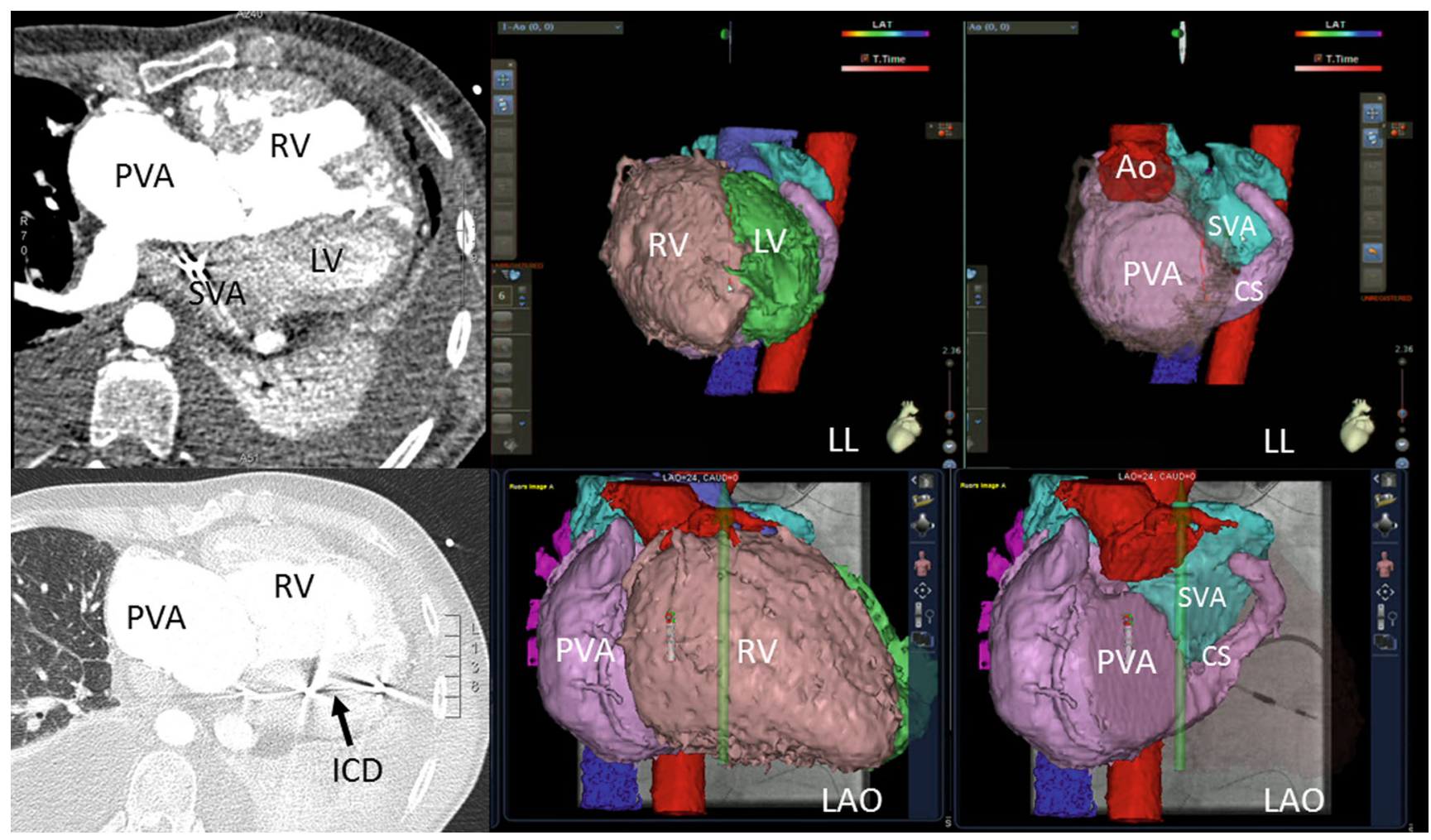

Abb. $2 \Delta$ Beispiel einer 3D-Rekonstruktion eines Kontrast-Computertomogramms bei Patient mit Transposition der großen Gefäße und Mustard-Operation. Unterschiedliche Kontrastmittelfüllung sowie Artefakt der Vorhof- und Implantierbarer-Kardioverter-Defibrillator(ICD)-Sonde im systemvenösen Kreislauf (SVA und LV) in Schichtaufnahmen gut sichtbar;3D-Konstruktionen demonstrieren Größe von rechtem Vorhof (RV) und pulmonalvenösem Vorhof (PVA); systemvenöser Vorhof (SVA) im Vergleich sehr klein; Ao Aorta, CS Koronarvenensinus, LAO links anterior oblique-Projektion, LL linkslateral, LV linker Ventrikel

jeweils in der Feldrichtung des äußeren Magnetfelds ausrichten. Die Richtung des äußeren Magnetfelds kann durch Positionsänderung der äußeren Permanentmagnete computerunterstützt erfolgen, was eine uneingeschränkte Beweglichkeit der Katheterspitze in alle Richtungen zur Folge hat. Mithilfe eines mechanischen Antriebs kann so der sehr flexible Katheter um jede Kurve oder Herzklappe ferngesteuert werden, wobei der Untersucher die Steuerung vom Kontrollraum durchführt (keine Strahlenbelastung für den Untersucher). Die Flexibilität der distalen Katheterspitze ist so hoch, dass bei Auftreffen auf ein Hindernis der Katheter abgeknickt wird, ohne dass eine Perforationsgefahr besteht. In der Kombination mit dem 3D-elektroanatomischen Mappingsystem $\mathrm{CARTO}^{\circledR}\left(\mathrm{CARTO}^{\circledR} \mathrm{RMT}^{\mathrm{R}}\right.$ Biosense Webster, Brüssel, Belgien) erlaubt die magnetische Navigation die direkte Integration von präprozeduralen Bildgebungsdaten und die Echtzeitdarstellung aller Katheter (Ablations- sowie alle weiteren diagnostischen Katheter) durch Bild-in-Bild-Darstellung (• Abb. 4; [9]). Eine vorbestehende Implantation eines Schrittmacher- oder Implantierbaren-Kardioverter-Defibrillator(ICD)-Systems ist keine Kontraindikation für den Einsatz der magnetischen Navigation. Um die durch das äußere Magnetfeld induzierte Magnetfunktion (Ventrikel-Ventrikel-inhibiert (VVI) 100/min) zu umgehen, wird für die Dauer der EPU die Funktion der Ventrikelsonde so programmiert, das sie keine Myokarderregung verursacht. Bei komplett schrittmacherabhängigen Patienten ohne Ersatzrhythmus muss zuvor eine temporäre Stimulationssonde platziert werden (wie auch bei allen anderen EPU-Prozeduren).

\section{Typische intraatriale Reentry- Tachykardie}

\section{Bei Vorhofseptumdefekt}

Während nichtkorrigierte Patienten mit Vorhofseptumdefekt (ASD) meist mit rechtsatrialen Tachykardien aufgrund der Vergrößerung des rechten Vorhofs (u. U. typisches isthmusabhängiges Vorhofflattern des rechten Vorhofs) auffällig werden, stehen bei operierten Patienten intraatriale Reentry-Tachykardie (IART) um den Septum-Patch und/oder die Atriotomienarbe im Vordergrund. Die Atriotomienarbe erlaubt z. B. die Entstehung eines geschützten Korridors, in dem die Kreiserregung in einer bevorzugten Richtung leitet und an der Einbzw. Austrittsstelle nahe der unteren oder oberen Hohlvene das restliche Vorhofmyokard aktiviert (Achter-Kreiserregung bzw. Figure-of-8-Reentry). Beide Anteile der Acht müssen unterbrochen werden, wobei die Unterbrechung an der "Taille“ innerhalb des geschützten 

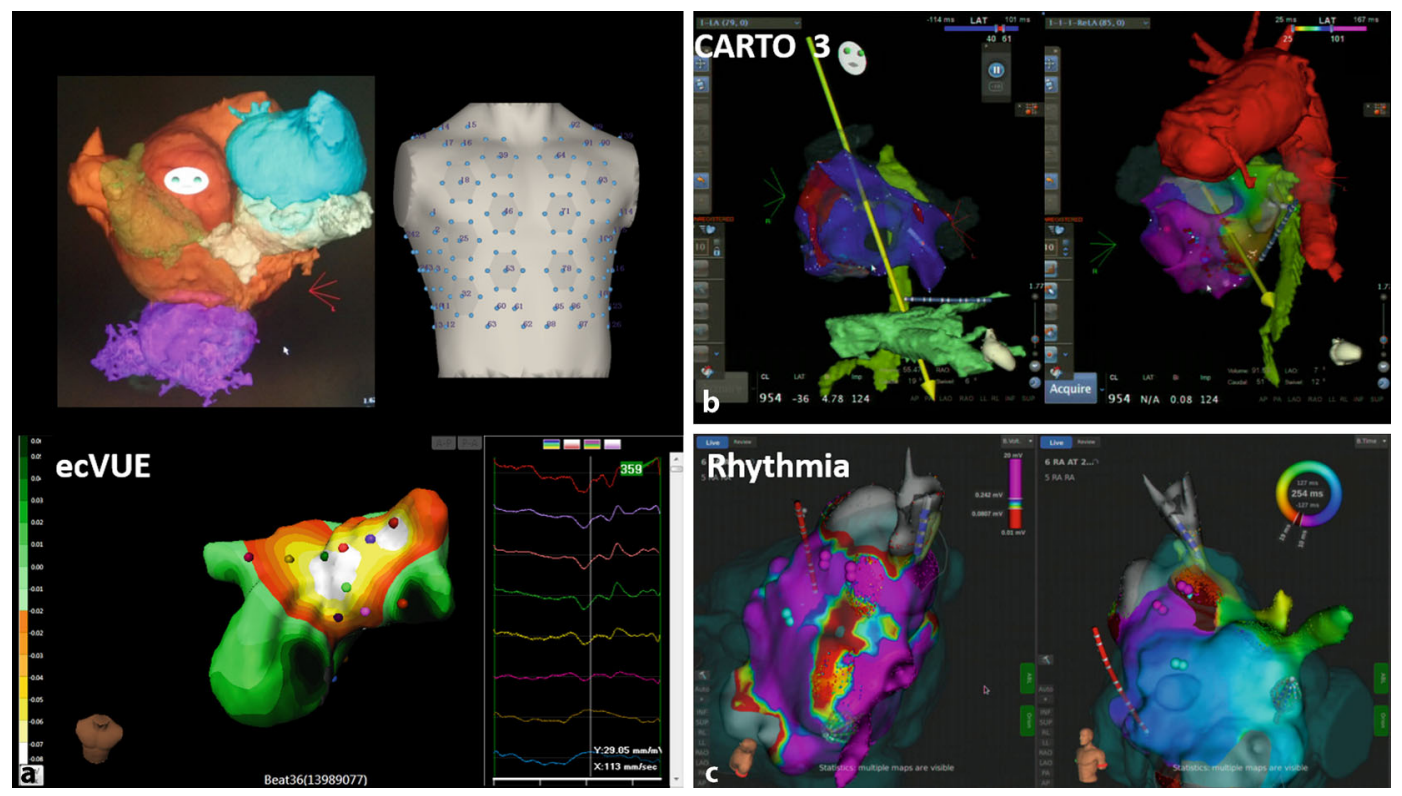

Abb. 3 ॥ Simultane vs. sequenzielle 3D-Mapping-Verfahren. a Exakte Lokalisierung von 252 Oberflächen-Elektrokardiogrammen im Nativ-Computertomogramm und Projektion auf 3D-Rekonstruktion der Vorhöfe (oder wahlweise der Kammern); Nichtinvasives Mapping erlaubt Darstellung jedes einzelnen Herzschlags über mehrere Stunden; anatomische Auflösung jedoch noch begrenzt; b 3D-Mapping und -Navigation durch elektroanatomisches CARTO-Mapping; Punkt-fürPunkt-Rekonstruktion des Reentry-Kreislaufs im Gegenuhrzeigersinn um Trikuspidalklappe („propagation map“ links) und Überprüfung des Ablationserfolgs im „re-map“ mit kompletter Ablationslinie am Boden des pulmonalvenösen Vorhofs (PVA) während konstanter Stimulation des systemvenösen Vorhofs (SVA); C Beispiel eines sequenziellen Mappings mithilfe Multielektroden-Basket-Katheters (Rhythmia ${ }^{\mathrm{TM}}$, Boston Scientific, Saint Paul/MN, USA) mit Voltage-Map und Aktivierungs-Map; Reentry-Kreislauf um kleines Narbenareal bei Patientin mit juxtapositionierten Vorhofohren

Korridors ebenfalls die Tachykardie unterbricht. Etwaige andere oder neukreierte Reentry-Substrate müssen sorgfältig gemappt und gegebenenfalls prophylaktisch unterbrochen werden (z. B. durch Isthmusblockade des rechten Vorhofs; [20]). Grundsätzlich ist bei Anlage von linearen Ablationen dieselbe Sorgfalt anzulegen und die Vollständigkeit der Ablationslinie durch Stimulationsmanöver und gegebenenfalls Remapping zu beweisen. Dies ist besonders hinsichtlich der schlechten hämodynamischen Toleranz bei langsamen Vorhoftachykardien mit Eins-zu-eins-Atrioventrikularüberleitung bei AHF-Patienten wichtig. Eine ausreichende Wartezeit nach erfolgreicher Ablation ist empfohlen, obwohl kein Konsensus bezüglich der Dauer der Wartezeit herrscht.

\section{Bei klassischer Fontan-Operation}

Patienten mit univentrikulärem Herz oder ähnlichen Vitien wurden früher häufig in Anlehnung an die ursprünglich von Fontan beschriebene Technik der direkten Verbindung von rechtem
Vorhof zur Pulmonalarterie (PA) unter Umgehung des rechten Ventrikels operiert. Während es eine große Anzahl von Modifikationen der ursprünglich beschriebenen Technik gibt, resultiert praktisch in allen Fällen als Langzeitkonsequenz eine massive Dilatation des rechten Vorhofs und hierdurch wiederum ein hohes Risiko für IART und fokale Tachykardien [5]. Wie auch bei anderen AHF-Substraten gehen die meisten atrialen Tachykardien (AT) auf Kreiserregungen um Narbenareale zurück, aber durch den konstant erhöhten Vorhofdruck ist die Ausprägung von diffuser Fibrose bei diesen Patient sehr ausgeprägt (• Abb. 1). Die größte Herausforderung besteht darin, ausreichenden Katheterkontakt zu erreichen und langfristig transmurale Läsionen anzulegen. Gelegentlich kann auch das Verständnis der zugrunde liegenden AT schwierig sein, da fokale AT nahe großer Narbenareale als Reentry-AT missverstanden werden können.

Insbesondere bei Patienten mit Double-inlet-(left)-Ventrikeln (DILV), bei denen der Chirurg durch Patch die rechtsseitige venöse Atrioventrikularklappe verschlossen hat, kann das alleinige Mapping des dilatierten rechten Vorhofs unvollständig sein. Um nicht das Reizleitungssystem zu beschädigen und um den Patch sicher zu fixieren, wird während der Operation routinemäßig ein kleiner Rest Vorhofmyokard zum Atrioventrikularklappenanulus auf der ventrikulären Seiten belassen. Der Koronarvenensinus wird ebenso häufig auf der ventrikulären Seite des Patches belassen. Konventionelles 3D-Mapping des rechten Vorhofs erlaubt deswegen nicht, die gesamte Kreiserregung um den Atrioventrikularklappenanulus darzustellen, weswegen das 3D-Mapping ergebnislos bleibt. Mithilfe der magnetischen Navigation gelang es schließlich den versteckten Anteil der Kreiserregung auf der ventrikulären Seite des Vorhofpatches aufzusuchen und erfolgreich zu ablatieren. Dabei wird der Katheter retrograd über die Aortenklappe, durch den Ventrikel und retrograd über die rechtsseitige Atrioventrikularklappe hinter den Patch gesteuert [28]. 


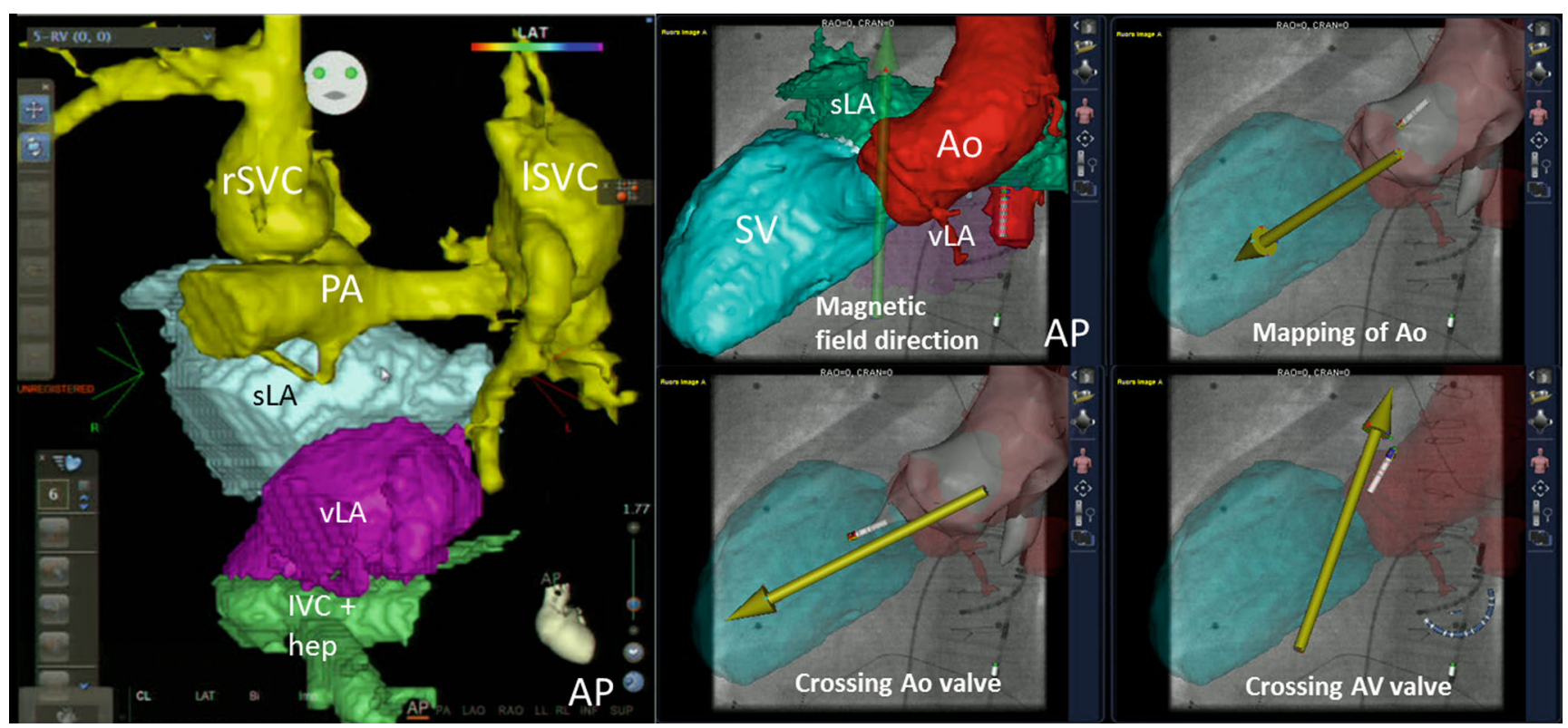

Abb. 4 ム Beispiel des retrograden Zugangs bei Patient mitlinksatrialem Isomerismus, Dextrokardie, Transposition dergroßen Gefäße (TGA) mit Ventrikelseptumdefekt (VSD) und Anastomose der Aorta (Ao) zum systemischen Ventrikel (SV) und bilateraler Glenn-Operation (bei beidseitiger V. cava superior [SVC]). Nach vorangegangenem, frustranem, konventionellem Ablationsversuch, nun retrograder Zugang mithilfe magnetischer Navigation mit CARTO ${ }^{\circledR}$ 3RMT (Biosense Webster, Brüssel, Belgien), Mapping der Aorta und Merging mit der 3D-Rekonstruktion der Magnetresonanztomographie, Passieren der Aortenklappe und Wechsel der magnetischen Navigationsrichtung (Pfeile) über die rechtsseitige Atrioventrikularklappe in den rechtsseitigen linken Vorhof, sequenzielles Mapping (• Abb. 3b) und erfolgreiche Ablation einer Vorhof-Reentry-Tachykardie. rSVC rechte obere Hohlvene, ISVC linke obere Hohlvene, sLA systemischer linker Vorhof, $v L A$ venoeser linker Vorhof, IVC inferiore Hohlvene, hep hepatische Gefaesse, $A P$ antero-posterior, $A V$ atrioventriculaer, $S V$ systemischer Ventrikel

\section{Typische Vorhoftachykardien}

\section{Nach totaler cavopulmonaler Konnektion}

Um den rechten Vorhof komplett zu umgehen und das systemvenöse Blut direkt den Pulmonalarterien zuzuführen, z. B. bei Trikuspidalklappenatresie, wird heutzutage eine totale cavopulmonale Konnektion (TCPC) durchgeführt. Dieser Tunnel besteht entweder komplett oder teilweise aus synthetischem Material (Goretex oder Dacron). Obwohl die Inzidenz von Vorhoftachykardien im Vergleich zur klassischen Fontan-Operation deutlich niedriger ist, kommen in diesem Kollektiv trotzdem Arrhythmien meist (aber nicht ausschließlich) in den nativen Vorhöfen vor. Insbesondere der native rechte Vorhof nach resultierender Umleitung der oberen und unteren Hohlvene ist konsekutiv so vernarbt, dass insbesondere Reentry um die rechtsseitige Atrioventrikularklappe häufig auftritt [1]. Fokale Tachykardien, Atrioventrikular-Reentry oder Atrio-
ventrikularknoten-Reentry-Tachykardien können ebenso auftreten und auch Twin-atrioventricular-nodal-Tachykardien sind beschrieben worden [25]. Zugang zu den nativen Vorhöfen kann durch trans-baffle oder trans-hepatische Punktion erreicht werden. Als Alternative bietet sich auch hier die magnetische Navigation an, die Zugang zu beiden nativen Vorhöfen auf retrogradem Wege erlaubt und analog zu der Erfahrung bei Patienten nach Mustard-SenningOperation durchgeführt wird. Wichtig ist es, nicht nur Tachykardieursprungsorte in den nativen Vorhöfen zu suchen, sondern auch an den Anastomosen der unteren und oberen Hohlvene sorgfältig zu mappen, insbesondere wenn mehr als eine Tachykardie induzierbar ist [29].

\section{Bei Ebstein-Anomalie}

Während Patienten mit Ebstein-Anomalie in jungen Jahren meist mit leitungsbahnassoziierten Arrhythmien auffallen (s. Beitrag von J. Hebe in diesem Heft), werden Vorhoftachykardien und auch
Vorhofflimmern im höheren Lebensalter häufiger beobachtet. Insbesondere nach chirurgischer Intervention an der Trikuspidalklappe und der dafür notwendigen Atriotomie treten bei diesen Patienten mit meist massiv dilatiertem rechten Vorhof anhaltende Tachykardien auf. Dies hat in den letzten Jahren zunehmend dazu geführt, dass intraoperativ zusätzliche Ablationslinien (z. B. durch Kryoenergie) angelegt werden, um diese IART zu unterdrücken und den rechten Vorhof zu kompartimentieren, wobei Erfolgsraten von bis zu $75 \%$ mit alleinigen Ablationen des rechten Vorhofs erreicht werden ([14]; s. Beitrag von Uemura et al. in diesem Heft).

Typische IART bei Ebstein-Anomalie treten, wie bei anderen Vitien mit massiver Dilatation des rechten Vorhofs v. a. um die Trikuspidalklappe, um die Atriotomienarbe oder aufgrund von inkompletten chirurgisch-applizierten Ablationslinien auf (iatrogene gap-assozierte IART). 


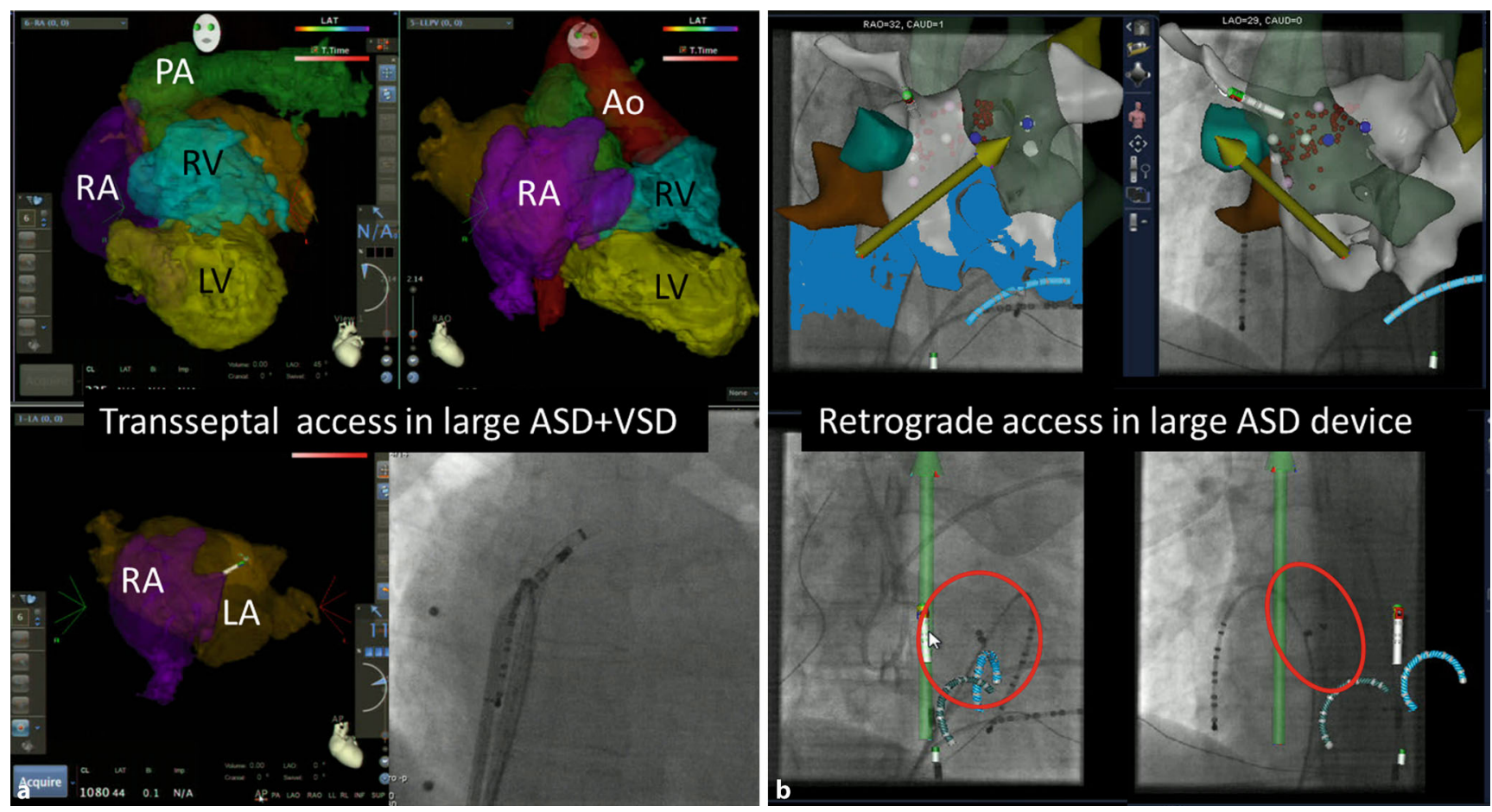

Abb. 5 A Beispiele von Vorhofflimmerablation bei Patienten mit AHF. a Patient mit großem Vorhofseptumdefekt (ASD) und Ventrikelseptumdefekt (VSD) ohne chirurgische Korrektur bei Criss-cross-Herz; obwohl großer ASD problemlosen Zugang zum linken Vorhof $(L A)$ erlaubt, ist aufgrund Vorhofgröße selbst Katheterablation von paroxysmalem Vorhofflimmern sehr schwierig; b Patientin mit sehr großem $(40 \mathrm{~mm})$ ASD-Okkluder; nachdem kein transseptaler Zugang erreichbar ist, wurde die Pulmonalvenenisolation retrograd mithilfe magnetischer Navigation durchgeführt. $R A$ rechter Vorhof, $P A$ Pulmonalarterie, $R V$ rechter Ventrikel, ASD Vorhofseptumdefekt

\section{Bei Fallot-Tetralogie}

Während ventrikuläre Tachykardien bei Patienten mit Fallot-Tetralogie (ToF) sicherlich die wichtigere Arrhythmie (s. Beitrag von Zeppenfeld in diesem Heft) darstellen, ist das Auftreten von Vorhoftachykardien doch ein signifikanter Marker für eine Verschlechterung der Gesamtsituation. Die Vergrößerung des rechten Vorhofs durch Volumenüberlastung z. B. bei inkompetenter Pulmonalarterienklappe, verursachen IART (peritrikuspidal, Periatriotomienarbe etc.) mit relevanter hämodynamischer Konsequenz bei vielen Patienten [20]. Detaillierte Analysen eines großen ToFKollektivs bestätigten diese Beobachtung anhand von MRT-Messungen von Vorhofgröße und Größe des rechten Ventrikels [4]. Speziell bei ToF-Patienten nach ICD-Implantation sollte auf gutes Sensing der Vorhofsonde geachtet werden, um eine sichere Differenzierung zwischen Vorhoftachykardie mit schneller Überleitung (mit dem Risiko einer inadäquaten Schockabgabe) und ventrikulärer Tachykardie zu erreichen.

\section{Bei Dextrotransposition der großen Gefäße}

In den 70er- bis 90er-Jahren geborenen Patienten mit Dextrotransposition der großen Gefäße (dTGA) wurde nach initialem Rashkind-Manöver typischerweise eine Vorhofumkehroperation nach Mustard (mit Baffle-Material aus Goretex oder Dacron) oder Senning (Perikard) durchgeführt, um die systemvenösen und pulmonalvenösen Vorhöfe dem jeweiligen Ventrikel zuzuleiten. Als Konsequenz ergibt sich ein rechter Ventrikel, der die Last des systemischen Kreislaufs tragen muss, was häufig zur Hypertrophie, Dilatation und in späteren Jahren zu ausgeprägter Herzinsuffizienz und ventrikulären Tachykardien führen kann. Vorhoftachykardien sind jedoch sehr häufig und beruhen zum einen auf Narbenareale, hervorgerufenen durch die multiplen Inzisionen, und zum anderen auf der aus der Belastung des rechten
Ventrikels resultierenden Vergrößerung des ehemaligen rechten Vorhofs, der nun Teil des pulmonalvenösen Vorhofs ist (-Abb.2). Eine kreisende Erregung um die Trikuspidalklappe ist das häufigste Tachykardiesubstrat, während IART um Narbenareale im pulmonalvenösen Vorhof seltener sind. Biatriale IART mit Aktivierung von pulmonalvenösem und systemvenösem Vorhof sind seltener. Fokale Tachykardien sind eher selten, aber insbesondere Vorhofflimmern wird bei diesen Patienten mit zunehmendem Durchschnittsalter mit steigender Wahrscheinlichkeit beobachtet. Zugang zum pulmonalvenösen Vorhof kann entweder durch Trans-baffle-Punktion [7] oder retrograd, transaortal und via rechtem Ventrikel erfolgen. Die Verwendung von konventionellen Ablationskathetern mit limitiertem und durch den Kurvenradius des Katheters bestimmtem Aktionsradius macht den Mapping- und Ablationsprozess bei retrogradem $\mathrm{Zu}$ gang sehr schwierig. Die Verwendung von 3D-Mapping-Verfahren sollte ein Standard bei diesen Prozeduren sein. 
Als Alternative, wenn vorhanden, bietet sich die magnetische Navigation an, bei der der Ablationskatheter mühelos retrograd in den pulmonalvenösen Vorhof gesteuert werden kann (• Abb. 4). Eine Reihe von Zentren haben über ihre erfolgreiche Technik mithilfe der magnetischen Navigation bei diesen komplexen Patienten berichtet und kommen übereinstimmend $\mathrm{zu}$ dem Ergebnis, dass so eine Trans-baffle-Punktion komplett vermieden werden kann [23, 29, 30]. Prozedurdauer und Gesamtstrahlenbelastung sind deutlich reduziert, was sowohl für die Patienten aber auch für die Untersucher von Bedeutung ist.

\section{Katheterablation von Vorhofflimmern}

Vorhofflimmern entwickelt sich häufig erst sehr spät nach chirurgischer Korrektur. Nachdem die Patienten nun jedoch zunehmend ein höheres Lebensalter erreichen, wird Vorhofflimmern zu einer neuen Arrhythmie für die es neuer Lösungsstrategien bedarf. Während bei Patienten mit normaler Herzanatomie eine elektrische Isolation der Pulmonalvenen meist ausreicht, haben AHFPatienten u. U. gänzlich andere Substrate, die Vorhofflimmern auslösen und aufrechterhalten (• Abb. 5). Die einzige Gruppe von AHF-Patienten, bei der in relevanter Zahl über erfolgreiche Ablationen des Vorhofflimmerns berichtet wurde, ist die Gruppe der Patienten mit ASD nach Vorhofseptumverschluss [15, $16,22]$. In vielen Fällen kann neben dem Verschlusssystem noch genügend Platz für den transseptalen Zugang gefunden werden, was normalerweise mithilfe transesophagealer oder intrakardialer Echokardiographie gut beurteilt werden kann. Sogar von direkten Punktionen durch das Verschlusssystem hindurch wurde berichtet ohne relevante Komplikationen durch den so entstandenen Shunt [22]. Als Alternative bei sehr großen Verschlusssystemen steht auch hier ein retrogrades Vorgehen mithilfe magnetischer Navigation zur Verfügung, das aber naturgemäß auf das alleinige Mapping und die Ablation mit dem Magnetkatheter limitiert ist [18]. Der Einsatz eines zirkumferenziellen (Lasso-)
Katheters ist dadurch ausgeschlossen, wodurch der Endpunkt der Pulmonalvenenisolation schwieriger $\mathrm{zu}$ beweisen ist.

Bei anderen Patientengruppen mit AHF und chirurgischer Korrektur gibt es außer Fallbeschreibungen noch keine Berichte über reproduzierbare Ablationsstrategien. Insbesondere bei Patienten mit dilatiertem, rechtem Vorhof scheint jedoch das Substrat v. a. rechtsatrial zu sein. Da die Konsequenz von Vorhofflimmern als Folge von unbehandelten, aber regelmäßigen Vorhoftachykardien angesehen werden kann, sollte alles daran gesetzt werden, dass der Sinusrhythmus erhalten und somit eine Progression zum Vorhofflimmern vermieden wird. Insbesondere ist auch auf eine ausreichende Antikoagulation hinzuweisen, da besonders in den großen Fontan-Vorhöfen Thromben entstehen können [17].

Im Einzelfall muss zum jetzigen Zeitpunkt eine Vorhofflimmerablation detailliert anhand der vorangegangen Operationen und dem zusätzlichen Fibrosemuster der Vorhöfe geplant und höchstwahrscheinlich mit deutlich erhöhter Rezidivrate im Vergleich zur Ablation in Patienten mit normal angelegtem Herz durchgeführt werden.

\section{Fazit für die Praxis}

- Katheterablation von regelmäßigen Vorhoftachykardien bei Patienten mit AHF werden heutzutage bei Einsatz der zur Verfügung stehenden Mapping- und Navigationstechniken mit guten Erfolgsaussichten durchgeführt.

- Insbesondere wenn der Zugang zur Zielregion durch Baffle-Material versperrt ist, hat sich die magnetische Navigation in Kombination mit 3DBildgebung als Alternative zur Transbaffle-Punktion bewährt.

- Planung und Durchführung eines Ablationseingriffs bei komplexen AHF bedarf der Zusammenarbeit mit Kollegen der Bildgebung, Anatomie, Intervention und Chirurgie sowie einer exzellenten Vor- und Nachbetreuung der Patienten im Rahmen von Expertenzentren.

\section{Korrespondenzadresse}

\section{S. Ernst, MD PhD FESC}

Department of Cardiology, Royal Brompton and Harefield NHS Foundation Trust Sydney Street, SW3 6NP London, UK s.ernst@rbht.nhs.uk

\section{Einhaltung ethischer Richtlinien}

Interessenkonflikt. S. Ernst hat Beraterverträge mit Biosense Webster \& Stereotaxis Inc. S. Yen Ho und K. McCarthy geben an, dass kein Interessenkonflikt besteht.

Dieser Beitrag beinhaltet keine von den Autoren durchgeführten Studien an Menschen oder Tieren.

Open Access. This article is distributed under the terms of the Creative Commons Attribution 4.0 International License (http://creativecommons.org/ licenses/by/4.0/), which permits unrestricted use, distribution, and reproduction in any medium, provided you give appropriate credit to the original author(s) and the source, provide a link to the Creative Commons license, and indicate if changes were made.

\section{Literatur}

1. Agnoletti G, Borghi A, Vignati G et al (2003) Fontan conversion to total cavopulmonary connection and arrhythmia ablation: clinical and functional results. Heart 89:193-198

2. Babu-Narayan SV, KilnerPJ, LiWetal (2006) Ventricular fibrosis suggested by cardiovascular magnetic resonance in adults with repaired tetralogy offallot and its relationship to adverse markers of clinical outcome. Circulation 113:405-413

3. Berul Cl, Van Hare GF, Kertesz NJ et al (2008) Results of a multicenter retrospective implantable cardioverter-defibrillator registry of pediatric and congenital heart disease patients. J Am Coll Cardiol 51:1685-1691

4. Bonello B, Kempny A, Uebing A et al (2013) Right atrial area and right ventricular outflow tract akinetic length predict sustained tachyarrhythmia in repaired tetralogy of Fallot. Int J Cardiol 168:3280-3286

5. Durongpisitkul K, Porter CJ, Cetta F et al (1998) Predictors of early- and late-onset supraventricular tachyarrhythmias after Fontan operation. Circulation 98:1099-1107

6. Ector J, De Buck S, Adams J et al (2005) Cardiac three-dimensional magnetic resonance imaging and fluoroscopy merging: a new approach for electroanatomic mapping to assist catheter ablation. Circulation 112:3769-3776

7. El-Said HG, Ing FF, Grifka RG et al (2000) 18-year experience with transseptal procedures through baffles, conduits, and other intra-atrial patches. Catheter Cardiovasc Interv 50:434-440

8. Ernst S, Babu-Narayan SV, Keegan J et al (2012) Remote-controlled magnetic navigation and ablation with 3D image integration as an alternative approach in patients with intra-atrial baffle anatomy. Circ Arrhythm Electrophysiol 5:131-139 
9. Ernst S, Chun JK, Koektuerk B et al (2009) Magnetic navigation and catheter ablation of right atrial ectopic tachycardia in the presence of a hemiazygos continuation: a magnetic navigation case using 3D electroanatomical mapping. J Cardiovasc Electrophysiol 20:99-102

10. Ernst S, Saenen J, Rydman R et al (2015) Utility of noninvasive arrhythmia mapping in patients with adult congenital heart disease. Card Electrophysiol Clin 7:117-123

11. Everett ITH, Lee KW, Wilson EE et al (2009) Safety profiles and lesion size of different radiofrequency ablation technologies: A comparison of large tip, open and closed irrigation catheters. J Cardiovasc Electrophysiol 20(3):325-335

12. Hebe J, Hansen P, Ouyang F et al (2000) Radiofrequency catheter ablation of tachycardia in patients with congenital heart disease. Pediatr Cardiol 21:557-575

13. Keegan J, Jhooti P, Babu-Narayan SV et al (2014) Improved respiratory efficiency of 3D late gadolinium enhancement imaging using the continuously adaptive windowing strategy (CLAWS). Magn Reson Med 71:1064-1074

14. Khositseth A, Danielson GK, Dearani JA et al (2004) Supraventricular tachyarrhythmias in Ebstein anomaly: management and outcome. J Thorac Cardiovasc Surg 128:826-833

15. Lakkireddy D, Rangisetty U, Prasad S et al (2008) Intracardiac echo-guided radiofrequency catheter ablation of atrial fibrillation in patients with atrial septal defect or patent foramen ovale repair: a feasibility, safety, and efficacy study. J Cardiovasc Electrophysiol 19:1137-1142

16. Li X, Wissner E, Kamioka M et al (2014) Safety and feasibility of transseptal puncture for atria fibrillation ablation in patients with atrial septal defect closure devices. Heart Rhythm 11:330-335

17. Mantziari L, Babu-Narayan SV, Suman-Horduna I et al (2013) Atrial arrhythmia after Fontan surgery leads to giant thrombus: opening Pandora's box. Int JCardiol 166:e23-24

18. Miyazaki S, Nault I, Haissaguerre M et al (2010) Atrial fibrillation ablation by aortic retrograde approach using a magnetic navigation system. J Cardiovasc Electrophysiol 21:455-457

19. Nakagawa $H$, Ikeda A, Sharma T et al (2012) Rapid high resolution electroanatomical mapping: evaluation of a new system in a canine atrial linear lesion model. Circ Arrhythm Electrophysiol 5:417-424

20. Nakagawa H, Shah N, Matsudaira K et al (2001) Characterization of reentrant circuit in macroreentrant right atrial tachycardia after surgical repair of congenital heart disease: isolated channels between scars allow "focal" ablation. Circulation 103:699-709

21. Paul T, Windhagen-Mahnert B, Kriebel $T$ et al (2001) Atrial reentrant tachycardia after surgery for congenital heart disease: endocardial mapping and radiofrequency catheter ablation using a novel, noncontact mapping system. Circulation 103:2266-2271

22. Santangeli $P$, Di Biase L, Burkhardt JD et al (2011) Transseptal access and atrial fibrillation ablation guided by intracardiac echocardiography in patients with atrial septal closure devices. Heart Rhythm 8:1669-1675

23. Schwagten $B$, Jordaens $L$, Witsenburg $M$ et al (2009) Initial experience with catheter ablation using remote magnetic navigation in adults with complex congenital heart disease and in small children. Pacing Clin Electrophysiol 32(Suppl 1):S198-S201
24. Sorensen TS, Therkildsen SV, Hansen OKet al (2002) Images in cardiovascular medicine. Total cavopulmonary connection: a virtual 3-dimensional fly-through. Circulation 105:E176-176

25. Suman-Horduna I, Babu-Narayan SV, Ueda A et al (2013) Magnetic navigation in adults with atrial isomerism (heterotaxy syndrome) and supraventricular arrhythmias. Europace 15:877-885

26. Tung R, Nakahara S, Maccabelli G et al (2011) Ultra high-density multipolar mapping with double ventricular access: a novel technique for ablation of ventricular tachycardia. J Cardiovasc Electrophysiol 22:49-56

27. Ueda A, Adachi I, Mccarthy KP et al (2013) Substrates of atrial arrhythmias: histological insights from patients with congenital heart disease. Int J Cardiol 168:2481-2486

28. Ueda A, Horduna I, Rubens Met al (2013) Reaching the ventricular aspect of the inferior isthmus in a Fontan patient using magnetic navigation. Heart Rhythm 10:1094-1095

29. Ueda A, Suman-Horduna I, Mantziari L et al (2013) Contemporary outcomes of supraventricular tachycardia ablation in congenital heart disease: a single-center experience in 116 patients. Circ Arrhythm Electrophysiol 6:606-613

30. Wu J, Pflaumer A, Deisenhofer I et al (2010) Mapping of atrial tachycardia by remote magnetic navigation in postoperative patients with congenital heart disease. J Cardiovasc Electrophysiol 21:751-759 